\title{
EL ACOMPAÑAMIENTO PEDAGÓGICO: UNA REVISION SISTEMATICA
}

\author{
THE PEDAGOGICAL SUPPORT: A SYSTEMATIC REVIEW
}

\section{Jeanette Milagros Abanto Zorrilla}

Maestro en Administración de la Educación, Universidad César Vallejo, Lima, Perú jabantoz@ucvvirtual.edu.pe ORCID(0000-0001-6092-8626)

https://scholar.google.es/citations?view_op=list_works\&hl=es\&user=RO5QpkMAAAAJ

\section{Maribel Rosales Armas}

Maestro en Gestión de los Servicios de Salud, Universidad César Vallejo, Lima, Perú, rrosalesarl@ucvvirtual.edu.pe ORCID (0000-0002-9194-1388)

https://scholar.google.es/citations?view_op=search_authors\&hl=es\&mauthors=maribel+ar mas+rosales\&btnG=

\section{Jaime Luna Sono}

Maestro en Gestión pública, Universidad César Vallejo, Lima, Perú jlunas@ucvvirtual.edu.pe ORCID (0000-0001-7374-8149)

https://scholar.google.es/citations?hl=es\&view_op=search_authors\&mauthors=jaime+luna + sono\&btnG $=$

\section{Revista Iberoamericana de la Educación Vol - Especial 12021 e-ISSN: 2737-632x}

Resumen: El presente artículo es un análisis desarrollado sobre el acompañamiento pedagógico como estrategia de formar a un docente. Se analizan diversas perspectivas de la forma como de acompañamiento en nuestro continente y otros. Basada en la metodología de Okoli et al. (2010) como soporte técnico de lo realizado, para lo cual se realizó una revisión bibliográfica sistemática con el objeto de documentarse sobre el problema, se efectuó la búsqueda en bases de datos y repositores de revistas científicas. del 2014 al 2021. La información procesada ha contribuido, de acuerdo con Cruz, Siles y Bustos (2021), de manera conceptual, antecedentes y diversas estrategias del acompañamiento pedagógico. Los resultados indicaron que el acompañamiento pedagógico es una estrategia importante que fortalece de manera efectiva el desempeño docente, asimismo, en la práctica pedagógica, 
se busca crear un aula en un ambiente de armonía y de comunicación afectiva entre el acompañante y acompañado para obtener mejores aprendizajes.

Palabras clave: acompañamiento pedagógico, practica pedagógico y formación docente.

Abstract: This article is an analysis developed on pedagogical accompaniment as a strategy to train a teacher. Various perspectives of the form of accompaniment in our continent and others are analyzed. Based on the methodology of Okoli et al. (2010) as technical support of what was done, for which a systematic bibliographic review was carried out in order to document the problem, a search was carried out in databases and repositories of scientific journals. from 2014 to 2021. The processed information has contributed, according to Cruz, Siles and Bustos (2021), in a conceptual way, antecedents and various strategies of pedagogical accompaniment. The results indicated that pedagogical accompaniment is an important strategy that effectively strengthens teaching performance, likewise, in pedagogical practice, it is sought to create a classroom in an environment of harmony and affective communication between the companion and accompanied to obtain better learning. Keywords: pedagogical accompaniment, pedagogical practice and teacher training.

\section{INTRODUCTION}

En el presente artículo se analiza el acompañamiento pedagógico como propuesta de formación profesional orientado a promover en los docentes de forma individual y colectiva, el fortalecimiento y mejora de su práctica docente en concordancia con las competencias del Marco del Buen Desempeño Docente (Minedu, 2012). Para Cruz et al. (2021) considera el 
acompañamiento pedagógico, importante para el fortalecimiento, del desempeño docente, asimismo, desde las fuentes responsables de la educación, lo asumen como un proceso de manera continua en todo su territorio. Cruz et al. (2021) establece que "lo fundamental, es brindar asesoría técnica a los docentes que permita reflexionar sobre su práctica pedagógica en el aula en un ambiente de armonía y de comunicación afectiva entre el acompañante y acompañado para obtener mejores aprendizajes”. (p.1)

García (2012, p.11) citado por Cruz et al. (2021, p.7), indicaron sobre el diccionario de la Real Academia de la Lengua, Acompañamiento significa "acción y efecto de acompañar, implica, participación en los sentimientos de los otros". Asimismo, definieron que "Este vocablo es una síntesis de necesidades, de sentimientos, de tareas y proyectos5 su desarrollo involucra a dos o más personas y a instituciones, que asumen un compromiso con la lógica del cambio personal, institucional y del contexto es decir la construcción de nuevas prácticas educativas, hacia un proyecto social y transformador". Para Agreda y Pérez (2020), definió al acompañamiento pedagógico una estrategia formativa ligada al intercambio de experiencias pedagógicas se inicia en el registro de situaciones observables, el diálogo reflexivo y los grupos de inter-aprendizaje entre docentes acompañados y acompañantes que permiten transformar sus prácticas docentes

Para Jabalera (2021, p.2), considera el acompañamiento pedagógico como "un instrumento que agrupa múltiples estrategias dirigidas al desarrollo de competencias docentes", indica también una falta de evidencias, de un empleo correcto para incidir en el desarrollo continuo de competencias básicas del docente en ejercicio. La estrategia utilizada por el autor fue el plan de acompañamiento pedagógico para conocer las competencias docentes y la 
forma como se transmite a los estudiantes. pero dichas estrategias no cuentan con el debido seguimiento después de conocer las oportunidades de mejora de cada docente. Este hecho nos invita a la reflexión sobre del rol que juegan dichas estrategias en el desarrollo profesional docente. Por otro lado, el estudio de Ferra (2021), examina el acompañamiento técnico-pedagógico en una plataforma virtual de aprendizaje para fortalecer la intervención docente en las aulas.

Almendras (2019) y Alzate \& Castañeda (2020) citados por González, Guanilo, Vegas, Cachicatari (2021), indicaron que la educación es el principal vehículo para la promoción del conocimiento y su difusión, basados en la formación de valores: sociales, culturales y colectivos que orientan al ser humano hacia la cooperación, la colaboración y el respeto por otros. Arboleda y Diaz (2017) citados por Gonzales et al. (2021) consideran que este lineamiento se basa en un modelo educativo que garantice un acompañamiento permanente de calidad a los profesores y repensar el concepto pedagógico, orientado a las necesidades del usuario, su desarrollo personal, social, económico y cultural colocándolo al nivel de los países desarrollados. Este acompañamiento puede producir beneficios para lograr una transformación significativa, requiriendo que las instituciones educativas sensibilizan sobre la necesidad por el cambio en sus aspectos administrativos y su dimensión pedagógica, fomentando nuevas visiones, ideas y prácticas enmarcadas en una dinámica de calidad ejecución.

Rodríguez, Medina, Tapia (202, p.2), consideran que el "docente es un factor fundamental en la calidad de la educación y responsable de lograr un aprendizaje auténtico en los estudiantes”. En su estudio, los autores, evidencian que en la mayoría de las instituciones carecen de procesos de 
inducción y que el profesorado novel enfrenta dificultades en la enseñanza, las relaciones interpersonales, la investigación y la vinculación con la sociedad. Consideran necesario que instituciones de educación superior implementen programas de inducción para el éxito de los nuevos maestros, mejorando su desempeño profesional y los aprendizajes de los estudiantes; así como reciban acompañamiento pedagógico para que se integre a la cultura organizacional de la institución. En el estudio de Siñani (2021), considero que existe una necesidad de incorporar la gestión educativa con las normas vigentes, así también encontró la falta de instrumentos de seguimiento y acompañamiento que sistematicen el desempeño profesional docente.

Del Valle (2020), realizaron un estudio sobre la percepción de estudiantes sobre el acompañamiento pedagógico virtual de un curso, determinando aprendizajes logrados para retroalimentar a la cátedra. Donde utilizaron las estrategias en el acompañamiento docente, expresadas a través de la evaluación colaborativa, evaluación de las actividades docentes, autoevaluación y devolución realizada. Rodríguez (2020), reflexionas sobre el rescate del acompañamiento pedagógico visto como una actitud abierta y creativa en la realidad universitaria. Abduraxmanovich (2020), considera el proceso de enseñanza profesional, donde la comunicación debe ser una estrategia continúa, asegurando la continuidad entre la formación y las actividades profesionales. Sobre el estudio de Valencia, Ledesma y Guevara (2020), explicaron que el acompañamiento pedagógico incide significativamente en la formación de los docentes, mediante las políticas de perfeccionamiento, prácticas y experiencias concretas de formación desarrolladas. 
El presente estudio encuentra su justificación en el acompañamiento docente el cual permitirá en la institución educativa, optimizar y reforzar los aspectos pedagógicos de los docentes y mejorar el aprendizaje de sus estudiantes, asimismo, la gestión supervisora dela institución, podrá tomar acciones de mejora pos-estudio.

En esta parte del estudio se plantean los antecedentes internacionales del estudio, empezando por: Pérez, Del Valle, Valles de Rojas, Lugo, Nava (2018), plantearon como objetivo analizar la supervisión efectuada en directores de educación media, para optimizar el acompañamiento pedagógico en las instituciones educativas. Estudio de paradigma positivista, muestra conformada por 24 directivos y 173 docentes de las unidades educativas oficiales del municipio Miranda, del Estado Zulia. Se empleó un cuestionario bajo escala tipo Likert, con cinco alternativas de respuestas, alcanzando un nivel de confiabilidad Alfa de Cronbach de 0.89 para directores y 0.98 para docentes. Los resultados indicaron que supervisión educativa, que permitió crear un buen clima de trabajo basado en la aplicación de una eficiente y eficaz forma de supervisión, Valencia, Ledesma y Guevara (2020, planteó como objetivo, determinar la incidencia del acompañamiento pedagógico en la formación de los docentes de la institución educativa de Perú. La investigación de nivel correlacional causal, muestra conformada por 65 docentes, la técnica utilizada fue la encuesta y los instrumentos utilizados para cada variable fueron cuestionarios. Los resultados indicaron que el acompañamiento pedagógico incide significativamente en la formación de los docentes de la Institución Educativa República de Bolivia de Lima - Perú; como también quedó demostrado que incide significativamente en las políticas de 
perfeccionamiento, prácticas y experiencias concretas de formación desarrolladas, producción académica.

\subsection{VARIABLE DE ESTUDIO}

En la tabla No 1, se muestra el tema estudiado, autor, el año en que se llevó a cabo a la investigación y las variables que tomaron en cuenta. se realizó una revisión bibliográfica sistemática con el objeto de documentarse sobre el problema, se efectuó la búsqueda en bases de datos y repositores de revistas científicas. del 2014 al 2021. La información procesada ha contribuido, de acuerdo con Cruz, Siles y Bustos (2021), de manera conceptual, antecedentes y diversas estrategias del acompañamiento pedagógico. Los estudios evaluados indican que el acompañamiento pedagógico, es un proceso muy importante en la educación del país y de cualquier lugar en el mundo.

Respecto al seguimiento del acompañamiento, traducido en supervisión, Pérez, Del Valle, Valles, Lugo and Nava (2018), consideran que supervisión educativa como parte del acompañamiento, es importante porque permite crear un agradable clima de trabajo, basado en la aplicación de una eficiente y eficaz forma de supervisión, para lograr el cambio de los patrones de conducta y de actuación de las personas comprometidas en el proceso educativo. Siñani (2021) propone el seguimiento para el mejoramiento de la gestión educativa, considerada por los docentes como un acto de relevancia que garantiza la excelencia académica.

Tabla No. 1. Variable 


\begin{tabular}{|c|c|c|c|c|}
\hline Tema & Autor & Año & País & Variables \\
\hline $\begin{array}{l}\text { Acompañamiento } \\
\text { pedagógico como } \\
\text { estrategia para } \\
\text { fortalecer el } \\
\text { desempeño docente } \\
\end{array}$ & $\begin{array}{c}\text { Cruz A., Siles D., } \\
\text { Bustos R. }\end{array}$ & 2021 & Nicaragua & $\begin{array}{l}\text { Acompañamiento } \\
\text { pedagógico } \\
\text { desempeño docente }\end{array}$ \\
\hline $\begin{array}{c}\text { Plan de } \\
\text { acompañamiento } \\
\text { pedagógico para } \\
\text { mejorar el } \\
\text { desempeño docente } \\
\text { en las instituciones } \\
\text { educativas del nivel } \\
\text { primario del } \\
\text { Municipio Consuelo }\end{array}$ & Jabalera S. & 2021 & $\begin{array}{c}\text { República } \\
\text { Dominica } \\
\text { na }\end{array}$ & $\begin{array}{l}\text { Plan de } \\
\text { acompañamiento } \\
\text { pedagógico } \\
\text { desempeño docente }\end{array}$ \\
\hline $\begin{array}{c}\text { Teaching } \\
\text { Accompaniment in } \\
\text { Colombia's } \\
\text { Official Educational } \\
\text { Institutions }\end{array}$ & $\begin{array}{c}\text { González R., } \\
\text { Guanilo S. Vegas J., } \\
\text { Cachicatari E. }\end{array}$ & 2021 & Colombia & $\begin{array}{l}\text { Teaching } \\
\text { Accompaniment }\end{array}$ \\
\hline $\begin{array}{c}\text { Teaching and } \\
\text { learning experience } \\
\text { with a virtual } \\
\text { classroom in the } \\
\text { field of pedagogical } \\
\text { support due to } \\
\text { Covid-19 }\end{array}$ & $\begin{array}{l}\text { Digión L., y } \\
\text { Álvarez M. }\end{array}$ & 2021 & México & $\begin{array}{l}\text { Soporte pedagógico } \\
\text { Enseñanza y } \\
\text { aprendizaje }\end{array}$ \\
\hline $\begin{array}{c}\text { Comunidades } \\
\text { virtuales e } \\
\text { innovación: } \\
\text { propuestas desde la } \\
\text { asesoría técnica } \\
\text { pedagógica en la } \\
\text { escuela } \\
\text { telesecundaria } \\
\text { Virtual }\end{array}$ & Ferra, G. & 2021 & Chile & $\begin{array}{l}\text { Asesoría técnica } \\
\text { pedagógica } \\
\text { Comunidades virtuales } \\
\text { e innovación:. }\end{array}$ \\
\hline $\begin{array}{l}\text { Supervisión y } \\
\text { acompañamiento } \\
\text { docente en la }\end{array}$ & Siñani & 2021 & Bolivia & $\begin{array}{l}\text { Supervisión } \\
\text { acompañamiento }\end{array}$ \\
\hline
\end{tabular}




\begin{tabular}{|c|c|c|c|c|}
\hline $\begin{array}{l}\text { Escuela Superior de } \\
\text { formación de } \\
\text { Maestros "Simón } \\
\text { Rodríguez", } \\
\text { Municipio } \\
\text { Quillacollo } \\
\end{array}$ & & & & \\
\hline $\begin{array}{c}\text { Relación entre el } \\
\text { acompañamiento } \\
\text { pedagógico y } \\
\text { práctica reflexiva }\end{array}$ & $\begin{array}{c}\text { Agreda A., Pérez } \\
\text { M. }\end{array}$ & 2020 & Argentina & $\begin{array}{l}\text { acompañamiento } \\
\text { pedagógico } \\
\text { práctica reflexiva }\end{array}$ \\
\hline $\begin{array}{l}\text { La Inducción Y El } \\
\text { Acompañamiento } \\
\text { Pedagógico Al } \\
\text { Profesorado Novel. } \\
\text { Una Oportunidad } \\
\text { Para Mejorar Su } \\
\text { Desempeño } \\
\text { Profesional } \\
\end{array}$ & $\begin{array}{c}\text { Rodríguez A. , } \\
\text { Medina M., Tapia } \\
\text { A. }\end{array}$ & 2020 & Ecuador & $\begin{array}{l}\text { La Inducción Y El } \\
\text { Acompañamiento } \\
\text { Pedagógico } \\
\text { Desempeño profesional }\end{array}$ \\
\hline $\begin{array}{l}\text { Percepción de las y } \\
\text { los estudiantes } \\
\text { sobre el } \\
\text { acompañamiento } \\
\text { pedagógico virtual } \\
\text { de la asignatura } \\
\text { Metodología de la } \\
\text { Investigación } \\
\text { Clínica y } \\
\text { Bioestadística } \\
\text { Aplicada en la } \\
\text { Carrera de Medicina } \\
\end{array}$ & Del Valle M. & 2020 & Argentina & $\begin{array}{l}\text { acompañamiento } \\
\text { pedagógico virtual }\end{array}$ \\
\hline $\begin{array}{c}\text { Intersubjetividad } \\
\text { Dialogica en el } \\
\text { Acompañamiento } \\
\text { del Docente } \\
\text { Instructor } \\
\text { Universitario }\end{array}$ & Rodríguez, María.. & 2020 & Venezuela & $\begin{array}{l}\text { Intersubjetividad } \\
\text { Dialogica } \\
\text { Acompañamiento Del } \\
\text { Docente }\end{array}$ \\
\hline $\begin{array}{c}\text { Modeling the } \\
\text { Pedagogical } \\
\text { Experience of } \\
\text { England as a Factor } \\
\text { of Improving the }\end{array}$ & $\begin{array}{c}\text { Abduraxmanovich, } \\
\text { J. U. }\end{array}$ & 2020 & $\begin{array}{l}\text { Uzbekista } \\
\mathrm{n}\end{array}$ & $\begin{array}{l}\text { Experiencia } \\
\text { pedagógica } \\
\text { Calidad de } \\
\text { entrenamiento }\end{array}$ \\
\hline
\end{tabular}




\begin{tabular}{|c|c|c|c|c|}
\hline $\begin{array}{c}\text { Quality of Training } \\
\text { Future Teachers }\end{array}$ & & & & \\
\hline $\begin{array}{l}\text { Acompañamiento } \\
\text { pedagógico en la } \\
\text { formación de los } \\
\text { docentes de la } \\
\text { Institución } \\
\text { Educativa } \\
\text { República de } \\
\text { Bolivia }\end{array}$ & $\begin{array}{l}\text { Valencia Romero, } \\
\text { A. M., Ledesma } \\
\text { Cuadros, M. J., \& } \\
\text { Guevara Gómez, H }\end{array}$ & 2020 & Perú & $\begin{array}{l}\text { Acompañamiento } \\
\text { pedagógico } \\
\text { Formación de los } \\
\text { docentes }\end{array}$ \\
\hline $\begin{array}{l}\text { Acompañamiento } \\
\text { pedagógico en la } \\
\text { formación de los } \\
\text { docentes de la } \\
\text { Institución } \\
\text { Educativa } \\
\text { República de } \\
\text { Bolivia }\end{array}$ & $\begin{array}{l}\text { Valencia Romero, } \\
\text { A. M., Ledesma } \\
\text { Cuadros, M. J., \& } \\
\text { Guevara Gómez, H }\end{array}$ & 2020 & Perú & $\begin{array}{l}\text { Acompañamiento } \\
\text { pedagógico } \\
\text { Formación de los } \\
\text { docentes }\end{array}$ \\
\hline $\begin{array}{c}\text { Desafíos De La } \\
\text { Escuela Media Hoy } \\
\text {. El } \\
\text { acompañamiento y } \\
\text { sostenimiento de las } \\
\text { trayectorias } \\
\text { escolares desde las } \\
\text { miradas de los } \\
\text { docentes } \\
\end{array}$ & $\begin{array}{c}\text { Arco M. / Belén } \\
\text { Godino, / Montiel Y } \\
\text { Luján }\end{array}$ & 2017 & Argentina & $\begin{array}{l}\text { El acompañamiento } \\
\text { Sostenimiento de las } \\
\text { trayectorias escolares }\end{array}$ \\
\hline $\begin{array}{l}\text { Gestión pedagógica } \\
\text { docente y ejecución } \\
\text { instrumental en } \\
\text { estudiantes de una } \\
\text { Escuela Superior de } \\
\text { Formación Artística }\end{array}$ & De la Cruz, E. & 2017 & Perú & $\begin{array}{l}\text { Gestión pedagógica } \\
\text { docente Ejecución } \\
\text { instrumental }\end{array}$ \\
\hline $\begin{array}{c}\text { Acompañamiento } \\
\text { pedagógico y } \\
\text { desempeño docente } \\
\text { en la } \\
\text { Institución } \\
\text { Educativa Manuel } \\
\text { Gonzales Prada, } \\
2016\end{array}$ & Huamaní F. & 2017 & Colombia & $\begin{array}{l}\text { Acompañamiento } \\
\text { pedagógico y } \\
\text { desempeño docente. }\end{array}$ \\
\hline
\end{tabular}




\begin{tabular}{|c|c|c|c|c|}
\hline $\begin{array}{c}\text { Methods of } \\
\text { Psychological and } \\
\text { Pedagogical } \\
\text { Accompaniment of } \\
\text { First-Year Students } \\
\text { in Process of } \\
\text { Adapting to } \\
\text { Learning at } \\
\text { University }\end{array}$ & Maralova et al. & 2016 & Rusia & $\begin{array}{l}\text { Métodos de } \\
\text { acompañamiento } \\
\text { psicológico y } \\
\text { pedagógico }\end{array}$ \\
\hline $\begin{array}{c}\text { Acompañamiento } \\
\text { Al Aula Como } \\
\text { Estrategia } \\
\text { Formadora Para El } \\
\text { Desarrollo } \\
\text { Profesional Docente }\end{array}$ & Mella & 2014 & Chile & $\begin{array}{l}\text { Acompañamiento Al } \\
\text { Aula } \\
\text { Estrategia Formadora } \\
\text { Para El Desarrollo } \\
\text { Profesional Docente }\end{array}$ \\
\hline
\end{tabular}

\section{MÉTODOS}

La investigación se fundamenta en la revisión y búsqueda documental, con parámetros de clasificación de las bases de datos como Scopus, Scielo, EBSCO y otros. Se realizó búsquedas de información, definidas por cada base de datos de revistas indexadas. El abordaje epistemológico y praxiológico de “Acompañamiento pedagógico" se basa en una síntesis de 18 artículos, encontradas, y también con el término de búsqueda "pedagogic accompaniment". Encontrándose propuestas educativas, protocolos de acompañamiento con diversos resultados. Con el fin de categorizar los artículos se clasificó los distintos tipos de investigación con sus respectivas unidades de observación e instrumentos (se listan las investigaciones en relación al año de aceptación en la tabla No. 1): 
Tabla No. 2. Métodos

\begin{tabular}{|c|c|c|c|}
\hline Tema & $\begin{array}{c}\text { Tipos de } \\
\text { Investigación }\end{array}$ & $\begin{array}{l}\text { Unidades de } \\
\text { Observación }\end{array}$ & $\begin{array}{c}\text { Métodos e } \\
\text { instrumento }\end{array}$ \\
\hline $\begin{array}{c}\text { Acompañamiento } \\
\text { pedagógico como estrategia } \\
\text { para fortalecer el } \\
\text { desempeño docente }\end{array}$ & Descriptiva & Docentes & $\begin{array}{l}\text { Método cualitativo } \\
\text { Entrevista al } \\
\text { docente }\end{array}$ \\
\hline $\begin{array}{c}\text { Plan de acompañamiento } \\
\text { pedagógico para mejorar el } \\
\text { desempeño docente en las } \\
\text { instituciones educativas del } \\
\text { nivel primario del } \\
\text { Municipio Consuelo }\end{array}$ & $\begin{array}{l}\text { metodología } \\
\text { cuantitativa, de } \\
\text { tipo diagnóstico- } \\
\text { propositiva }\end{array}$ & $\begin{array}{l}20 \\
\text { docentes }\end{array}$ & $\begin{array}{l}\text { Cuestionario } \\
\text { aplicado a los } \\
\text { docentes }\end{array}$ \\
\hline $\begin{array}{l}\text { Teaching Accompaniment } \\
\text { in Colombia's } \\
\text { Official Educational } \\
\text { Institutions }\end{array}$ & $\begin{array}{l}\text { Metodología } \\
\text { cuantitativa } \\
\text { Correlacional }\end{array}$ & 341 profesores & $\begin{array}{l}\text { Cuestionario Con } \\
36 \text { Reactivos } \\
\text { (Likert Escala } \\
\text { Tipográfica) }\end{array}$ \\
\hline $\begin{array}{c}\text { La Inducción Y El } \\
\text { Acompañamiento } \\
\text { Pedagógico Al Profesorado } \\
\text { Novel. Una Oportunidad } \\
\text { Para Mejorar Su } \\
\text { Desempeño Profesional }\end{array}$ & $\begin{array}{l}\text { revisión } \\
\text { bibliográfica } \\
\text { sistemática }\end{array}$ & $\begin{array}{l}\text { bases de datos y } \\
\text { repositores de } \\
\text { revistas } \\
\text { científicas }\end{array}$ & $\begin{array}{l}\text { Revisión } \\
\text { bibliográfica y } \\
\text { documental }\end{array}$ \\
\hline $\begin{array}{l}\text { Teaching and learning } \\
\text { experience with a virtual } \\
\text { classroom in the field of } \\
\text { pedagogical support due to } \\
\text { Covid-19 }\end{array}$ & $\begin{array}{l}\text { Investigación } \\
\text { descriptiva }\end{array}$ & 104 alumnos & $\begin{array}{l}\text { Cuestionarios y } \\
\text { chats }\end{array}$ \\
\hline $\begin{array}{l}\text { Comunidades virtuales e } \\
\text { innovación: propuestas } \\
\text { desde la asesoría técnica } \\
\text { pedagógica en la escuela } \\
\text { telesecundaria Virtual }\end{array}$ & $\begin{array}{l}\text { investigación- } \\
\text { acción, n } \\
\text { análisis de } \\
\text { carácter } \\
\text { cualitativo, } \\
\text { continuo y } \\
\text { sistemático }\end{array}$ & cinco docentes, & $\begin{array}{l}\text { Entrevistas, foros } \\
\text { de discusión, } \\
\text { reuniones }\end{array}$ \\
\hline $\begin{array}{c}\text { Supervisión y } \\
\text { acompañamiento docente en } \\
\text { la Escuela Superior de } \\
\text { formación de Maestros }\end{array}$ & $\begin{array}{l}\text { enfoque mixto } \\
\text { (cualitativo } \\
\text { cuantitativo }\end{array}$ & 26 docentes & Entrevista \\
\hline
\end{tabular}




\begin{tabular}{|c|c|c|c|}
\hline $\begin{array}{l}\text { "Simón Rodríguez", } \\
\text { Municipio Quillacollo }\end{array}$ & & & \\
\hline $\begin{array}{c}\text { Relación entre el } \\
\text { acompañamiento } \\
\text { pedagógico y práctica } \\
\text { reflexiva }\end{array}$ & $\begin{array}{l}\text { investigación } \\
\text { descriptiva no } \\
\text { experimental } \\
\text { con diseño } \\
\text { correlacional } \\
\text { explicativo }\end{array}$ & 150 docentes & Cuestionarios \\
\hline $\begin{array}{l}\text { Percepción de las y los } \\
\text { estudiantes sobre el } \\
\text { acompañamiento } \\
\text { pedagógico virtual de la } \\
\text { asignatura Metodología de } \\
\text { la Investigación Clínica y } \\
\text { Bioestadística Aplicada en } \\
\text { la Carrera de Medicina }\end{array}$ & $\begin{array}{l}\text { Investigación } \\
\text { descriptiva }\end{array}$ & $\begin{array}{l}4 \text { docentes } \\
17 \text { estudiantes }\end{array}$ & Cuestionarios \\
\hline $\begin{array}{l}\text { Intersubjetividad Dialogica } \\
\text { en el Acompañamiento del } \\
\text { Docente Instructor } \\
\text { Universitario }\end{array}$ & $\begin{array}{l}\text { Enfoque } \\
\text { cualitativa bajo } \\
\text { la hermenéutica } \\
\text { como método }\end{array}$ & $\begin{array}{l}3 \text { sujetos actores } \\
\text { sociales }\end{array}$ & $\begin{array}{l}\text { Entrevista a } \\
\text { profundidad }\end{array}$ \\
\hline $\begin{array}{l}\text { Modeling the Pedagogical } \\
\text { Experience of England as a } \\
\text { Factor of Improving the } \\
\text { Quality of Training Future } \\
\text { Teachers }\end{array}$ & $\begin{array}{l}\text { método de } \\
\text { observación, el } \\
\text { método } \\
\text { estadístico y el } \\
\text { análisis de } \\
\text { contenido. }\end{array}$ & $\begin{array}{l}360 \text { estudiantes } \\
\text { de último año de } \\
\text { universidades } \\
\text { pedagógicas de } \\
\text { nuestra } \\
\text { república }\end{array}$ & Entrevistas \\
\hline $\begin{array}{l}\text { Educational Supervision As } \\
\text { Pedagogical } \\
\text { Accompaniment Conducted } \\
\text { By Secondary Education } \\
\text { Directors }\end{array}$ & $\begin{array}{l}\text { paradigma } \\
\text { positivista, } \\
\text { diseño no } \\
\text { experimental, } \\
\text { transeccional }\end{array}$ & $\begin{array}{l}24 \text { directivos y } \\
173 \text { docentes de } \\
\text { las unidades } \\
\text { educativas } \\
\text { oficiales del } \\
\text { municipio } \\
\text { Miranda, del } \\
\text { Estado Zulia }\end{array}$ & $\begin{array}{l}\text { Cuestionario bajo } \\
\text { escala tipo Likert, }\end{array}$ \\
\hline $\begin{array}{c}\text { Acompañamiento } \\
\text { pedagógico en la formación } \\
\text { de los docentes de la } \\
\text { Institución Educativa } \\
\text { República de Bolivia }\end{array}$ & $\begin{array}{l}\text { enfoque } \\
\text { cuantitativo, tipo } \\
\text { descriptivo, } \\
\text { diseño no } \\
\text { experimental, } \\
\text { nivel }\end{array}$ & 65 docentes & Cuestionarios. \\
\hline
\end{tabular}




\begin{tabular}{|c|c|c|c|}
\hline & $\begin{array}{l}\text { correlacional } \\
\text { causal, de corte } \\
\text { transversal }\end{array}$ & & \\
\hline $\begin{array}{c}\text { Desafíos De La Escuela } \\
\text { Media Hoy . El } \\
\text { acompañamiento y } \\
\text { sostenimiento de las } \\
\text { trayectorias escolares desde } \\
\text { las miradas de los docentes }\end{array}$ & $\begin{array}{l}\text { Enfoque } \\
\text { cuantitativo. } \\
\text { Diseño } \\
\text { experimental }\end{array}$ & Docentes & Cuestionarios \\
\hline $\begin{array}{c}\text { Gestión pedagógica } \\
\text { docente y ejecución } \\
\text { instrumental en estudiantes } \\
\text { de una Escuela Superior de } \\
\text { Formación Artística }\end{array}$ & $\begin{array}{l}\text { nivel descriptivo } \\
\text { correlacional }\end{array}$ & 30 estudiantes & Cuestionarios \\
\hline $\begin{array}{c}\text { Acompañamiento } \\
\text { pedagógico y desempeño } \\
\text { docente en la } \\
\text { Institución Educativa } \\
\text { Manuel Gonzales Prada, } \\
2016\end{array}$ & $\begin{array}{l}\text { Investigación } \\
\text { correlacional }\end{array}$ & 85 profesores & $\begin{array}{l}\text { Cuestionarios con } \\
15 \text { reactivos cada } \\
\text { uno }\end{array}$ \\
\hline $\begin{array}{l}\text { Methods of Psychological } \\
\text { and Pedagogical } \\
\text { Accompaniment of First- } \\
\text { Year Students in Process of } \\
\text { Adapting to Learning at } \\
\text { University }\end{array}$ & $\begin{array}{l}\text { Investigación } \\
\text { descriptiva }\end{array}$ & $\begin{array}{l}350 \text { maestros } \\
450 \text { estudiantes }\end{array}$ & Cuestionarios \\
\hline $\begin{array}{c}\text { Acompañamiento Al Aula } \\
\text { Como Estrategia Formadora } \\
\text { Para El Desarrollo } \\
\text { Profesional Docente }\end{array}$ & $\begin{array}{l}\text { estudio de caso } \\
\text { con enfoque } \\
\text { cualitativo de } \\
\text { tipo descriptivo }\end{array}$ & 60 profesores & $\begin{array}{l}\text { Instrumento del } \\
\text { Ministerio de } \\
\text { Educación } \\
\text { "Cuestionario para } \\
\text { la autoevaluación } \\
\text { institucional" }\end{array}$ \\
\hline
\end{tabular}

Se puede apreciar en la tabla No 2, los diversos tipos de metodología, de diversos enfoques. Asimismo, los sujetos de análisis e instrumentos que se utilizaron en cada investigación. Se observa que la variable analizada está presente en distintos países especialmente latinos, con diferentes propuestas, para mejorar la enseñanza, es común en todos los estudios. 


\section{RESULTADOS}

En base a los estudios recopilados, se exponen en la Tabla 3, los resultados, donde se muestra el aporte de cada uno de ellos a la comunidad científica.

Tabla 3. Resultados

\begin{tabular}{|c|c|}
\hline Tema & Resultados \\
\hline $\begin{array}{l}\text { Acompañamiento pedagógico } \\
\text { como estrategia para fortalecer } \\
\text { el desempeño docente }\end{array}$ & $\begin{array}{l}\text { Permitió reflexionar sobre la práctica pedagógica en el aula } \\
\text { creando un ambiente de armonía y de comunicación afectiva } \\
\text { entre el acompañante y acompañado para obtener mejores } \\
\text { aprendizajes }\end{array}$ \\
\hline $\begin{array}{l}\text { Plan de acompañamiento } \\
\text { pedagógico para mejorar el } \\
\text { desempeño docente en las } \\
\text { instituciones educativas del } \\
\text { nivel primario del Municipio } \\
\text { Consuelo }\end{array}$ & $\begin{array}{l}\text { Los docentes de las escuelas mostraron un bajo nivel de } \\
\text { desempeño, el mismo está relacionado por la falta de un } \\
\text { efectivo programa de acompañamiento pedagógico }\end{array}$ \\
\hline $\begin{array}{l}\text { Teaching Accompaniment in } \\
\text { Colombia's } \\
\text { Official Educational } \\
\text { Institutions }\end{array}$ & $\begin{array}{l}\text { Existencia de una relación entre los principios de calidad } \\
\text { y acompañamiento docente en la enseñanza oficial } \\
\text { instituciones de la ciudad de Montería }\end{array}$ \\
\hline $\begin{array}{l}\text { La Inducción Y El } \\
\text { Acompañamiento Pedagógico } \\
\text { Al Profesorado Novel. Una } \\
\text { Oportunidad Para Mejorar Su } \\
\text { Desempeño Profesional }\end{array}$ & $\begin{array}{l}\text { Promueve que las instituciones de educación superior } \\
\text { implementen programas de inducción para el éxito de los } \\
\text { nuevos maestros, mejoran su desempeño profesional y los } \\
\text { aprendizajes de los estudiantes; así como reciban } \\
\text { acompañamiento pedagógico para que se integre a la } \\
\text { cultura organizacional de la institución }\end{array}$ \\
\hline $\begin{array}{l}\text { Teaching and learning } \\
\text { experience with a virtual } \\
\text { classroom in the field of } \\
\text { pedagogical support due to } \\
\text { Covid-19 }\end{array}$ & $\begin{array}{l}\text { La experiencia de clase virtual, se considera un } \\
\text { componente altamente aprovechable para el trabajo } \\
\text { docente en la búsqueda de un estudiante autónomo } \\
\text { respecto al logro de metas en su proceso de enseñanza- } \\
\text { aprendizaje, como lo muestra la experiencia realizada. }\end{array}$ \\
\hline
\end{tabular}




\begin{tabular}{|c|c|}
\hline $\begin{array}{c}\text { Comunidades virtuales e } \\
\text { innovación: propuestas desde } \\
\text { la asesoría técnica pedagógica } \\
\text { en la escuela telesecundaria } \\
\text { Virtual }\end{array}$ & $\begin{array}{l}\text { La propuesta de innovación, a través, de la asesoría } \\
\text { técnica pedagógica en la escuela telesecundaria Virtual, } \\
\text { considera que el trabajo colaborativo en el diseño de } \\
\text { situaciones didácticas con miras a fortalecer la } \\
\text { intervención docente en las aulas. }\end{array}$ \\
\hline $\begin{array}{l}\text { Supervisión y acompañamiento } \\
\text { docente en la Escuela Superior } \\
\text { de formación de Maestros } \\
\text { "Simón Rodríguez", } \\
\text { Municipio Quillacollo }\end{array}$ & $\begin{array}{l}\text { Propone la necesidad de involucrar la gestión educativa } \\
\text { con la normativa vigente y la falta de instrumentos de } \\
\text { seguimiento y acompañamiento que sistematicen el } \\
\text { desempeño profesional docente. Por tal motivo se } \\
\text { propuso una guía de acompañamiento y seguimiento para } \\
\text { el mejoramiento de la gestión educativa, considerada por } \\
\text { los docentes como un acto de relevancia que garantiza la } \\
\text { excelencia académica. }\end{array}$ \\
\hline $\begin{array}{l}\text { Relación entre el } \\
\text { acompañamiento pedagógico y } \\
\text { práctica reflexiva }\end{array}$ & $\begin{array}{l}\text { La práctica reflexiva que desarrollan los docentes durante } \\
\text { su ejercicio e interacción con sus estudiantes ponen en } \\
\text { práctica un conjunto de actividades de análisis y } \\
\text { valoración antes, durante y después de esas acciones } \\
\text { pedagógicas }\end{array}$ \\
\hline $\begin{array}{l}\text { Percepción de las y los } \\
\text { estudiantes sobre el } \\
\text { acompañamiento pedagógico } \\
\text { virtual de la asignatura } \\
\text { Metodología de la } \\
\text { Investigación Clínica y } \\
\text { Bioestadística Aplicada en la } \\
\text { Carrera de Medicina }\end{array}$ & $\begin{array}{l}\text { Durante este acompañamiento se compartió una mirada } \\
\text { integral del proceso educativo, donde docentes y } \\
\text { estudiantes pudieron llevar a cabo una construcción } \\
\text { dialógica virtual satisfactoria. }\end{array}$ \\
\hline $\begin{array}{l}\text { Intersubjetividad Dialogica en } \\
\text { el Acompañamiento del } \\
\text { Docente Instructor } \\
\text { Universitario }\end{array}$ & $\begin{array}{l}\text { Los hallazgos más significativos fueron recurrentes en } \\
\text { torno a la comunicación, confianza, co-construcción del } \\
\text { conocimiento y supervisión. Se reflexiona acerca del } \\
\text { rescate del acompañamiento pedagógico cuando se } \\
\text { comprende la condición humana que emerge como una } \\
\text { actitud abierta y creativa en la realidad universitaria }\end{array}$ \\
\hline $\begin{array}{l}\text { Modeling the Pedagogical } \\
\text { Experience of England as a } \\
\text { Factor of Improving the } \\
\text { Quality of Training Future } \\
\text { Teachers }\end{array}$ & $\begin{array}{l}\text { Propone formar una competencia comunicativa } \\
\text { profesional en lengua extranjera, el dominio de una } \\
\text { lengua extranjera en la comunicación cotidiana sin tener } \\
\text { en cuenta las especificidades de una profesión. }\end{array}$ \\
\hline $\begin{array}{l}\text { Educational Supervision As } \\
\text { Pedagogical Accompaniment } \\
\text { Conducted By Secondary } \\
\text { Education Directors } \\
\end{array}$ & $\begin{array}{l}\text { La supervisión educativa como acompañamiento } \\
\text { pedagógico de los directores, permite crear un buen clima } \\
\text { de trabajo basado en la aplicación de una eficiente y } \\
\text { eficaz forma de supervisión. }\end{array}$ \\
\hline
\end{tabular}




\begin{tabular}{|c|c|}
\hline $\begin{array}{l}\text { Acompañamiento pedagógico } \\
\text { en la formación de los docentes } \\
\text { de la Institución Educativa } \\
\text { República de Bolivia }\end{array}$ & $\begin{array}{l}\text { Los resultados indicaron que el acompañamiento } \\
\text { pedagógico incide significativamente en la formación de } \\
\text { los docentes de la Institución Educativa. quedó } \\
\text { demostrado que incide significativamente en las políticas } \\
\text { de perfeccionamiento, prácticas y experiencias concretas } \\
\text { de formación desarrolladas, producción académica. }\end{array}$ \\
\hline $\begin{array}{l}\text { Desafíos De La Escuela Media } \\
\text { Hoy . El acompañamiento y } \\
\text { sostenimiento de las } \\
\text { trayectorias escolares desde las } \\
\text { miradas de los docentes }\end{array}$ & $\begin{array}{l}\text { Propuesta formativa elaborada por un equipo de } \\
\text { profesores de una universidad y de un instituto de } \\
\text { formación docente de la ciudad de Villa Mercedes. } \\
\text { <Logrando un acompañamiento y sostenimiento de las } \\
\text { trayectorias escolares, eficaz. }\end{array}$ \\
\hline $\begin{array}{l}\text { Gestión pedagógica docente y } \\
\text { ejecución instrumental en } \\
\text { estudiantes de una Escuela } \\
\text { Superior de Formación } \\
\text { Artística } \\
\end{array}$ & $\begin{array}{l}\text { Evidenciaron una correlación de Spearman un nivel de } \\
\text { significancia del } 5 \% \text {, de Rho = } 0.843 \text {; existe una relación } \\
\text { directa entre la gestión pedagógica de los docentes y la } \\
\text { ejecución instrumental. }\end{array}$ \\
\hline $\begin{array}{l}\text { Acompañamiento pedagógico } \\
\text { y desempeño docente en la } \\
\text { Institución Educativa Manuel } \\
\text { Gonzales Prada, } 2016\end{array}$ & $\begin{array}{l}\text { Se obtuvo una correlación Rho de Spearman } \\
\text { considerando el error al } 5 \% \text { de la relación resultó de Rho } \\
=0,815^{* *} \text { con la significancia de } p_{\text {_ }} \text { value }=0.000< \\
0,05 \text { se concluyó que existe correlación directa y } \\
\text { significativa entre el acompañamiento pedagógico y el } \\
\text { desempeño docente en la organización educativa Manuel } \\
\text { Gonzales Prada de la Ugel } 02,2016 \text {. }\end{array}$ \\
\hline $\begin{array}{l}\text { Methods of Psychological and } \\
\text { Pedagogical Accompaniment } \\
\text { of First-Year Students in } \\
\text { Process of Adapting to } \\
\text { Learning at University }\end{array}$ & $\begin{array}{l}\text { El conjunto de métodos identificados de acompañamiento } \\
\text { psicológico y pedagógico asegura la formación de } \\
\text { pensamiento reflexivo de los estudiantes; formación de la } \\
\text { actitud constructiva social y profesional interacción con } \\
\text { otros; Dominar las habilidades y destrezas de la } \\
\text { estabilidad psicológica en situaciones de riesgo. }\end{array}$ \\
\hline $\begin{array}{c}\text { Acompañamiento Al Aula } \\
\text { Como Estrategia Formadora } \\
\text { Para El Desarrollo Profesional } \\
\text { Docente }\end{array}$ & $\begin{array}{l}\text { Muestran que el diseño de un modelo de } \\
\text { acompañamiento al aula basado en la reflexión } \\
\text { permanente del profesorado para fortalecer el desarrollo } \\
\text { profesional docente. }\end{array}$ \\
\hline
\end{tabular}

La información obtenida a partir de los artículos muestra el estado del acompañamiento docente, en sus diferentes perspectivas, lo que lleva a una reflexión y el trabajo por hacer, especialmente en la educación de nuestro país en sus diferentes niveles. 


\section{CONCLUSIONES}

El acompañamiento pedagógico es una estrategia importante que fortalece de manera efectiva el desempeño docente, asimismo, en la práctica pedagógica, se busca crear un aula en un ambiente de armonía y de comunicación afectiva entre el acompañante y acompañado para obtener mejores aprendizajes. La realización de un Plan de acompañamiento pedagógico, se convierte en una estrategia importante para mejorar el desempeño docente en las instituciones educativas.

Los principios de calidad, en el acompañamiento docente en la enseñanza oficial, son también importantes a ser tomadas en cuenta. Asimismo, los programas de inducción para mejorar el desempeño profesional e integrarlo a la cultura organizacional de la institución. La innovación en una propuesta de asesoría técnica pedagógica, fortalece la intervención docente en las aulas.

Existen debilidades en la falta de instrumentos de seguimiento y acompañamiento que sistematicen el desempeño profesional docente. En la busca de excelencia académica, es importante construir una guía de acompañamiento y seguimiento para el mejoramiento de la gestión educativa. Los estudios observados corresponden en su mayoría al enfoque cuantitativo, de nivel descriptivo y correlacional, en menos proporción de enfoque cualitativo y mixto. Los instrumentos utilizados en su mayoría son cuestionarios y por otro lado entrevistas a profundidad. Las investigaciones se basan en estadísticas de hechos reales. La población y muestra de las investigaciones consideradas, está basada en directores, docentes y estudiantes, que han sido considerados como sujetos de estudio, lo cual se observa y se determina los actores involucrados en el acompañamiento docente. 


\section{REFERENCIAS BIBLIOGRÁFICAS}

Abduraxmanovich, J. U. (2020). Modeling the Pedagogical Experience of England as a Factor of Improving the Quality of Training Future Teachers. International Journal of Psychosocial Rehabilitation, 24(04). DOI: 10.37200/IJPR/V24I4/PR2020481

Agreda A., Perez M. (2020), Relación entre el acompañamiento pedagógico y práctica reflexiva. Espacios en blanco Revista de Educación Nro. 30 vol.2jul/dic.2020, pp.219-232Universidad Nacional del Centro de la Provincia de Buenos Aires, Tandil, Argentina. DOI: https://doi.org/10.37177/UNICEN/EB30-273

Almendras Fernández, M. R. (2019). Gestión por competencia en el desarrollo de las habilidades directivas de las Instituciones Educativas de la Red N. ${ }^{\circ} 5$ Ventanilla, 2019.

Alzate-Ortiz, F. A., \& Castañeda-Patiño, J. C. (2020). Mediación pedagógica: Clave de una educación humanizante y transformadora. Una mirada desde la estética y la comunicación. Revista Electrónica Educare, 24(1), 411-424.

Arboleda, M. L. O., \& Díaz, R. R. G. (2017). El carácter axiológico del proceso educativo en Colombia. Centros: Revista Científica Universitaria, 6(2), 1-17.

Arco M., Belén G, Montiel y Luján (2017) Desafíos de la escuela media hoy el acompañamiento y sostenimiento de las trayectorias escolares desde las miradas de los docentes. Revista Mexicana de Investigación Educativa, 2017, vol. 22, núm. 72, pp. 159-177 (ISSN: 14056666) 
Cruz A., Siles D., Bustos R. (2021), Acompañamiento pedagógico como estrategia para fortalecer el desempeño docente Revista Educativa de Nicaragua. $\mathrm{N}^{\circ}$, Año 01. Abr.-Sept. 2021.

Digión L., y Álvarez M. (2021) Teaching and learning experience with a virtual classroom in the field of pedagogical support due to Covid-19 Revista apertura, Volumen 13, número 1, pp. 20-35 Universidad de Guadalajara http://doi.org/10.32870/Ap.v13n1.1957

Del Valle M. (2020) Percepción de las y los estudiantes sobre el acompañamiento pedagógico virtual de la asignatura Metodología de la Investigación Clínica y Bioestadística Aplicada en la Carrera de Medicina de marzo a julio 2020. Revista Argentina de educación médica. Vol. 9 No 4 diciembre, 7-13, 2020

De la Cruz, E. (2017). Gestión pedagógica docente y ejecución instrumental en estudiantes de una Escuela Superior de Formación Artística. Propósitos y Representaciones, 5(2), $321 \quad$ - $357 . \quad$ doi: http:// dx.doi.org/10.20511/pyr2017.v5n2.175

Ferra-Torres, Gerson. (2021). Virtual communities and innovation: proposals from the technical pedagogical advisory in telesecondary school. IE Revista de Investigación Educativa de la REDIECH. 12. 10.33010/ie_rie_rediech.v12i0.1102.

García, D. (2007). El acompañamiento, proceso indispensable para el fortalecimiento de la calidad de la educación.Ponencia en el Seminiario Capacitación de Técnicos y Docentes del Nivel Medio. Santo Domingo: Intec.

González R., Guanilo S. Vegas J., Cachicatari E. (2021) Teaching Accompaniment in Colombia's 
Official Educational Institutions TEM Journal. Volume 10, Issue 1, Pages 376-383, ISSN 2217-8309, DOI: 10.18421/TEM101-47 https://doi.org/10.18421/TEM101-47

González R., Guanilo S. Vegas J., Cachicatari E. (2021) Teaching Accompaniment in Colombia'sOfficial Educational Institutions TEM Journal. Volume 10, Issue 1, Pages 376-383, ISSN 2217-8309, DOI: 10.18421/TEM101-47 https://doi.org/10.18421/TEM101-47

Huamani Arredondo (2017), Pedagogical accompaniment and teaching performance at the Educational Institute Manuel Gonzales Prada, 2016”. Universidad Alas Peruanas

Jabalera S. (2021) Plan de acompañamiento pedagógico para mejorar el desempeño docente en las instituciones educativas del nivel primario del Municipio Consuelo UCE Ciencia. Revista de postgrado. Vol. 9(1), 2021

Maralova et al (2016) Methods of Psychological and Pedagogical Accompaniment of First-Year Students in Process of Adapting to Learning at University. International Journal Of Environmental \& Science Education 2016, VOL. 11, NO. 17, 10569-10579

Minedu. (2012). Marco del buen desempeño docente. Lima, Perú

Mella (2014) Acompañamiento Al Aula Como Estrategia Formadora Para El Desarrollo Profesional Docente. Universidad Católica de Temuco, Escuela de Posgrado. Chile 
Okoli, C. y Schabram, K. (2010). A guide to conducting a systematic literature review of information systems research. Sprouts: Working Papers on Information Systems.10(26).

Perez-Medina, J., Del Valle-Giraldoth, D., Valles de Rojas, M., Lugo-Cueva, L., and Nava, N. (2018). Educational Supervision As Pedagogical Accompaniment Conducted By Secondary Education Directors. Revista Panorama, 12(23), 85-102. DOI: http://dx.doi.org/10.15765/pnrm.v12i23

Rodríguez A. , Medina M., Tapia A. (2021), La Inducción Y El Acompañamiento Pedagógico Al Profesorado Novel. Una Oportunidad Para Mejorar Su Desempeño Profesional. Revista Educare. Volumen $24 \mathrm{~N}^{\mathrm{o}} 3$ septiembre-diciembre 2020

Rodríguez M. (2020). Intersubjetividad Dialogica En El Acompañamiento Del Docente Instructor Universitario. Areté. 6. 217-237. Fecha de recepción: 27 febrero 2020 Fecha de aceptación: 4 abril 2020

Siñani (2021) Supervisión y acompañamiento docente en la Escuela Superior de ormación de Maestros “Simón Rodríguez”, Municipio Quillacollo Revista Frank Tamayo, Vol. 3, No. 6 | Enero - abril 2021 | Páginas 10 - 27 ISSN: 2710 - 088X / ISSN-L: 2710 - 088X

Valencia A. M., Ledesma, M. J., \& Guevara, H. E. (2020). Acompañamiento pedagógico en la formación de los docentes de la Institución Educativa República de Bolivia, Perú. Alpha Centauri, 1(2), 15-29. https://doi.org/10.47422/ac.v1i2.9 